\title{
Empirically-derived food patterns and the risk of total mortality and cardiovascular events in the PREDIMED study
}

\section{YCLNU-D-14-00319R2}

Miguel A Martínez-González ${ }^{1,2}$, Itziar Zazpe ${ }^{2,3}$, Cristina Razquin ${ }^{1}$, Ana Sánchez-Tainta ${ }^{1,2}$, Dolores Corella ${ }^{2,4}$, Jordi Salas-Salvadó2,5, Estefanía Toledo ${ }^{1,2}$, Emilio Ros ${ }^{2,6}$, Miguel Ángel Muñoz $^{7,8}$, Javier Recondo ${ }^{9}$, Enrique Gómez-Gracia, ${ }^{2,10}$, Miquel Fiol ${ }^{2,11}$, José Lapetra ${ }^{2,12}$, Pilar Buil-Cosiales $^{1,2,13}$, Lluis Serra-Majem ${ }^{2,14}$, Xavier Pinto ${ }^{2,15}$, Helmut Schroeder ${ }^{2,16}$, Josep A Tur ${ }^{2,17}$, José V. Sorli ${ }^{2,4}$, Rosa M Lamuela-Raventós ${ }^{2,18}$, Ramón Estruch ${ }^{2,6}$ FOR THE PREDIMED GROUP

${ }^{1}$ Department of Preventive Medicine and Public Health, University of Navarra, Pamplona ${ }^{2}$ CIBER Fisiopatologia de la Obesidad y Nutricion (CIBERobn) and PREDIMED Network (RD 06/0045) from Instituto de Salud Carlos III (ISCIII) Spanish Government.

${ }^{3}$ Department of Nutrition and Food Sciences and Physiology, University of Navarra, Pamplona.

${ }^{4}$ Department of Preventive Medicine, University of Valencia, Valencia

${ }^{5}$ Human Nutrition Department, IISPV, Universitat Rovira i Virgili, Reus

${ }^{6}$ Institut d'Investigacions Biomediques August Pi Sunyer (IDIBAPS), Hospital Clinic, University of Barcelona, Barcelona;

${ }^{7}$ Unitat de Suport a la Recerca, División de Atención Primaria de Salud, Institut Català de la Salut e IDIAP-Jordi Gol, Barcelona, Spain

${ }^{8}$ Departamento de Pediatría, Obstetricia, Ginecología y Medicina Preventiva, Universitat Autònoma de Barcelona, Barcelona, Spain

${ }^{9}$ Department of Cardiology, University Hospital of Alava, Vitoria

${ }^{10}$ Department of Preventive Medicine, University of Malaga, Malaga 
${ }^{11}$ Institute of Health Sciences IUNICS, University of Balearic Islands, and Hospital Son Espases, Palma de Mallorca

${ }^{12}$ Department of Family Medicine, Primary Care Division of Sevilla, San Pablo Health Center, Sevilla

${ }^{13}$ Servicio Navarro de Salud-Osasunbidea, Pamplona

${ }^{14}$ Department of Clinical Sciences, University of Las Palmas de Gran Canaria, Las Palmas

${ }^{15}$ Lipids and Vascular Risk Unit, Internal Medicine, Hospital Universitario de Bellvitge, Hospitalet de Llobregat, Barcelona

${ }^{16}$ Cardiovascular and Nutrition Research Group, Institut de Recerca Hospital del Mar-IMIM, Barcelona

${ }^{17}$ Department of Fundamental Biology \& Health Sciences, University of Balearic Islands

${ }^{18}$ Department of Nutrition and Food Science, School of Pharmacy, XaRTA, INSA, University of Barcelona, Barcelona

Correspondence and reprints: Prof. M. A. Martínez-González, MD, MPH, PhD. Department of Preventive Medicine and Public Health, Facultad de Medicina- Clínica Universidad de Navarra, Universidad de Navarra, Irunlarrea 1, 31080 Pamplona, Navarra, Spain. E-mail: mamartinez@unav.es. Tel: +34 948425600 Ext: 6463. Fax: + 34948425649. 


\section{ABSTRACT}

2 Background and aims: There is little evidence on post hoc- derived dietary patterns (DP)

3 and all-cause mortality in Southern-European populations. Furthermore, the potential effect

4 modification of a DP by a nutritional intervention has not been sufficiently assessed. We assessed the association between a posteriori defined baseline major DP and total mortality or

6 cardiovascular events within each of the three arms of a large primary prevention trial

7 (PREDIMED) where participants were randomized to two active interventions with

8 Mediterranean-type diets or to a control group (allocated to a low-fat diet).

9 Design: We followed-up 7216 participants for a median of 4.3 years. A validated 137 -item food-frequency questionnaire was administered. Baseline DP were ascertained through factor analysis based on 34 predefined groups. Cox regression models were used to estimate multivariable-adjusted hazard ratios (HR) for cardiovascular disease (CVD) or mortality across quartiles of DP within each of the three arms of the trial.

Results: We identified two major baseline DP: the first DP was rich in red and processed meats, alcohol, refined grains and whole dairy products and was labeled Western Dietary Pattern (WDP). The second DP corresponded to a "Mediterranean-type" dietary pattern (MDP). During follow-up, 328 participants died. After controlling for potential confounders, higher baseline adherence to the MDP was associated with lower risk of CVD (adjusted HR for fourth vs. first quartile: $0.52 ; 95 \% \mathrm{CI}$ (Confidence Interval): $0.36,0.74$; p-trend $<0.001$ ) and all-cause mortality (adjusted HR: 0.53; 95\% CI: 0.38, 0.75; p-trend $<0.001$ ), regardless of

21 the allocated arm of the trial. An increasing mortality rate was found across increasing quartiles of the WDP in the control group (allocated to a low-fat diet), though the linear trend was not statistically significant $(\mathrm{p}=0.098)$.

CONCLUSIONS: Higher adherence to an empirically-derived MDP at baseline was associated with a reduced risk of CVD and mortality in the PREDIMED trial regardless of the

* CVD: Cardiovascular Disease, DP:Dietary Pattern, EVOO: Extra Virgin Olive Oil, FFQ: Food Frequency Questionnaire, IPW: Inverse Probability Weighting, MeDiet:Mediterranean Diet, MDP: Mediterranean Dietary Pattern, WDP: Western Dietary Pattern. 
allocated arm. The WDP was not associated with higher risk of mortality or cardiovascular events.

\section{INTRODUCTION}

The main causes of death in developed countries are cancer and cardiovascular disease (CVD)*, chronic conditions in which prevention by lifestyle, particularly a healthy diet, plays an important role.

In the context of overall dietary patterns (DP), many epidemiological studies have examined the health benefits of an a priori defined Mediterranean diet (MeDiet) and found consistent evidence that individuals who better adhere to this DP have a healthier ageing and a longer life span [1, 2].

In the last decade there has been growing interest in assessing the relationships between diet and disease through the study of whole DP instead of focusing on single nutrients or foods [3]. The approach consisting in collecting food data and using them afterwards to identify the DP actually followed by the study subjects is known as the a posteriori approach (post hoc). It detects DP empirically derived from available data using principal component analysis or factor analysis [46]. Though several studies have assessed the relationship between a posteriori DP and various health outcomes [7], including all-cause mortality [7-10], there is little evidence on post hoc DP and mortality in Southern European populations [11]. An interesting issue that has not been explored is whether the adverse effects of a DP can be modified by a nutritional intervention. We evaluated the association between a posteriori defined major DP and CVD incidence or total mortality in each of the three arms of a large randomized trial testing an intervention with a Mediterranean-type diets for the primary prevention of CVD (the PREDIMED study). Participants in the PREDIMED trial were randomized to two active interventions with Mediterranean-type diets or to a control group (allocated to a low-fat diet). We assessed 
whether this intervention was able to modify the association between baseline DP and two outcomes: all-cause mortality and the risk of cardiovascular events.

\section{METHODS}

\section{Subjects}

The PREDIMED (PREvención con DIeta MEDiterránea) trial (ISRCTN35739639) is a multicenter, randomized, controlled, parallel group, primary prevention trial conducted in Spain to assess the effects of MeDiet on major cardiovascular events. The study protocol has been published elsewhere $[12,13]$ and full details are available at www.predimed.es. Between October 2003 and June 2009 we recruited 7,447 high-risk participants and randomly assigned them to one of three dietary interventions: two MeDiets supplemented with either extra virgin olive oil (EVOO) or mixed nuts and a control group (low-fat diet). Participants in the two MeDiet groups received either extra-virgin olive oil (to consume $50 \mathrm{~g} / \mathrm{d}$ ) or mixed nuts per day ( $15 \mathrm{~g} / \mathrm{d}$ walnuts, $7.5 \mathrm{~g} / \mathrm{d}$ hazelnuts, and $7.5 \mathrm{~g} / \mathrm{d}$ almonds $)$ at no cost. They received instructions directed to upscale the traditional MeDiet 14-item score, including 1) the use of olive oil for cooking and dressing; 2) increased consumption of vegetables, nuts, and fish products; 3) consumption of white meat instead of red or processed meat; 4) preparation of home-made sauce by simmering tomato, garlic, onion and aromatic herbs with olive oil to dress vegetables, pasta, rice and other dishes; and 5) for alcohol drinkers, to follow a moderate pattern of red wine consumption. Participants allocated to the control group received small nonfood gifts and were advised to reduce all types of fat and were given written recommendations according to American Heart Association guidelines. No total calorie restriction was advised, nor was physical activity promoted in any of the three groups. The primary endpoint for the main trial was a composite of cardiovascular events. Total mortality was also used as a secondary outcome. All participants provided written informed consent to a protocol approved by the institutional review boards of the recruiting centers. 
Eligible participants were community-dwelling men aged 55-80 years and women aged 60-80 years without previous CVD who fulfilled at least one of the following criteria: type-2 diabetes or 3 or more cardiovascular risk factors, namely smoking, hypertension, dyslipidemia, overweight (BMI $\geq 25 \mathrm{~kg} / \mathrm{m}^{2}$ ) or a family history of premature CVD. For the present study we excluded 231 participants, 79 of them because their baseline food-frequency questionnaires (FFQ) were missing and 152 who displayed out-of-range total energy intake ( $<500$ or $>3500 \mathrm{kcal} / \mathrm{d}$ in women or $<800$ or $>4000 \mathrm{kcal} / \mathrm{d}$ in men) [14]. Consequently, the final sample size included 7,216 participants.

\section{Dietary assessment}

Data on dietary intake were collected at baseline with a semi-quantitative 137 -item validated FFQ [15]. For each item, a typical portion size was included, and consumption frequencies were registered in 9 categories that ranged from "never or almost never" to " $\geq 6$ times/day". Daily food consumption was estimated by multiplying the portion size of each food item by its consumption frequency. Energy and nutrient intake were derived using a computer program based on available information in Spanish food composition tables.

\section{Assessment of non- dietary variables}

Several questionnaires were used at baseline examination to collect sociodemographic data, lifestyle variables, history of illnesses, and medication use. A validated questionnaire [16] was used to collect information on physical activity.

\section{Ascertainment of mortality}

During follow-up dietitians delivered the nutritional intervention with quarterly individual visits and quarterly group sessions [13]. The questionnaires and examinations carried out at baseline were repeatedly administered every year to all participants. Besides, once a year a team of medical doctors reviewed medical records to collect information on the main outcomes, both in primary care centers and hospitals. Also, yearly inquiries were made of the 
100 National Death Index. Considering all the sources of information, we are reasonably confident

101 that ascertainment of mortality outcomes was complete. When a death was identified using

102 any of these primary sources, medical records were requested where the patient had been

103 cared for and submitted to the end-point adjudication committee for assignment of the cause

104 of death. This committee was blinded with respect to the intervention group and the food

105 habits of participants.

\section{Statistical analyses}

107 The 137 food items included in the FFQ were grouped into 34 predefined food categories. A

108 principal component analysis was applied to these 34 categories in order to identify a reduced

109 number of factors that could explain the maximum proportion of the variance from the

110 original groups. Food groups with absolute loading $>0.29$ were considered relevant

111 components of the identified patterns (Table 1).

112 The score for each participant was calculated by summing up the consumption of each food

113 group weighted by each factor score obtained in the factor analysis. The resulting quantitative

114 scores were categorized into quartiles. The Scree plot and the criteria of eigenvalues $>2$ were

115 used to select factors. We selected 2 factors that accounted for $13 \%$ of the total variance.

116 Baseline characteristics and nutritional habits of participants according to their quartiles of

117 adherence to both selected factors (dietary patterns) were analyzed (tables 2 and 3 ) and p

118 values for linear trend tests were calculated for each variable. Nutritional variables were log-

119 transformed to calculate the $\mathrm{p}$ values for linear trend.

120 We used Cox regression models with length of follow-up since randomization for the trial as

121 the primary time variable. The exposure time was calculated as the time elapsed between

122 recruitment and the date of death for deceased participants, the last study visit, or the last

123 recorded clinical event of participants still alive. Hazard ratios (HR) with $95 \%$ confidence

124 intervals (CI) for the three upper quartiles compared to the lowest quartile of each DP were 
125 calculated. In multivariable model 1, potential confounders included as covariates were sex, 126 age (continuous), intervention group and recruitment center. We constructed a second model

127 (multivariable model 2) that also included as covariates smoking status (never, former or

128 current smoker), baseline BMI ( $\mathrm{kg} / \mathrm{m}^{2}$, continuous), physical activity during leisure time

129 (METs min/day, continuous), baseline self-reported hypertension, hypercholesterolemia,

130 diabetes, history of previous depression and educational level (three categories). We

131 conducted analyses stratified by each of the three arms of the trial. We repeated the main

132 analyses adjusting also for total energy intake ( $\mathrm{kcal} /$ day, continuous). Tests of linear trend

133 across successive quartiles of adherence to each of the two food patterns were calculated.

134 In order to assess the effects of the intervention across the quartiles of baseline adherence to

135 each DP, a new variable was created combining the joint exposure to the quartile of adherence

136 to the studied DP and the intervention. This cross-classification was conducted with the aim

137 of assessing whether the intervention was able to modify the association between baseline

138 adherence to DP and the risk of death or cardiovascular events. We used inverse probability

139 weighting (IPW) not only to control for baseline confounding but also to estimate the absolute

140 risks according to the joint classification of participants in 12 groups by both quartile of the

141 dietary pattern and intervention arm allocated in the trial [17]. To calculate the weights we

142 used as predictors baseline values of age, sex, smoking habit, BMI, physical activity,

143 hypertension, hypercholesterolemia, diabetes, educational level and history of depression.

144 This method allows analyzing observational studies (the effects of baseline adherence to DP

145 in our case) in a way similar to a randomized trial, under the assumption that all relevant

146 confounders are included in the computation of weights. We estimated the absolute risks of

147 mortality (or cardiovascular events) for each combined category of the DP (quartiles) and the

148 intervention group ( 3 categories), using the weighted pseudo-population obtained with the

149 IPW procedure. Relative risks were also calculated for upper quartiles of each DP using as 
150 reference the first quartile of the DP, within each intervention group and the $\mathrm{p}$ for linear trend

151 was calculated.

152 We used STATA version 12.0 (StataCorp) for all analyses.

\section{RESULTS}

154 The mean ( \pm SD) age of the 7,216 participants was 67.0 (6.2) y. Participants were followed-

155 up for an average of $4.3 \mathrm{y}$. During this period 328 deaths were recorded; the mean age at

156 death was $73.8(6.9)$.

157 Factor analysis revealed two major DP. Absolute factor loadings $>0.29$ for each DP are

158 presented in Table 1. The first factor was characterized by a high consumption of high fat

159 processed meats and red meats, alcohol, refined grains, canned fish, whole-fat dairy products,

160 sauces, eggs, processed meals, commercial bakery and chocolates, whereas consumption of

161 low-fat dairy products was inversely loaded. We labeled it as "Western Dietary Pattern

162 (WDP)".

163 The second factor was loaded with vegetables, EVOO, walnuts, oily fish and canned fish,

164 fruits, other nuts, whole-wheat bread, white fish and low fat dairy products. In addition, it was

165 defined by a low consumption (negative loadings) of refined grains and of other olive oils

166 different from EVOO. We labeled this second factor as Mediterranean-type dietary pattern

167 (MDP).

168 The baseline characteristics of participants by quartiles of the WDP and the MDP are shown

169 in Tables 2 and 3 respectively. Subjects with a higher adherence to the WDP were more

170 likely to be men, current smokers and were more physically active. They also showed higher

171 total energy intake and significantly higher intakes of fat, except for monounsaturated fatty

172 acids, and also greater consumption of most food groups with the exception of vegetables and 
Table 1. Factor loadings for the two major dietary patterns in the PREDIMED study

Alcohol

0.45

Red meat

0.45

Refined grains

0.40

$-0.30$

Canned fish/Seafood

0.38

0.38

Sauces

0.33

Processed meal

0.32

Whole dairy products

0.31

Eggs

0.30

Commercial bakery

0.29

Chocolates

0.29

Low fat dairy products

$-0.33$

Olive oil (not extra-virgin)

$-0.39$

White fish

0.33

Whole grain bread

0.35

Nuts (not walnut)

0.36

Fruit

0.36

Oily fish

0.39

Walnuts

0.40

Extra-virgin olive oil

0.47

Vegetables

0.51 
Table 2. Baseline characteristics of the 7216 participants according to quartiles of adherence to Western Dietary Pattern (WDP)

\section{Men (\%)}

Smoking status

$$
\text { Former smokers (\%) }
$$

Current smokers (\%)

Diabetes $(\%)$

Hypertension (\%)

Age (y)

Weight $(\mathrm{kg})$

BMI $\left(\mathrm{kg} / \mathrm{m}^{2}\right)$

Physical activity during leisure time (METs-min/day)

Total energy intake (kcal/d)

Carbohydrate intake (\% total energy)

Protein intake ( $\%$ total energy)

Fat intake ( $\%$ total energy)

Monounsaturated fatty acid intake (\% total energy)

Polyunsaturated fatty acid intake (\% total energy)

Saturated fatty acid intake ( $\%$ total energy)

Alcohol intake (g/d)

Vegetables $(\mathrm{g} / \mathrm{d})$

Fruits (g/d)

Whole-fat dairy products $(\mathrm{g} / \mathrm{d})$

Low-fat dairy products (g/d)

\begin{tabular}{|c|c|c|c|c|}
\hline $\begin{array}{c}\text { WDP1 } \\
(n=1804)\end{array}$ & $\begin{array}{c}\text { WDP2 } \\
(n=1804)\end{array}$ & $\begin{array}{c}\text { WDP3 } \\
(n=1804)\end{array}$ & $\begin{array}{c}\text { WDP4 } \\
(n=1804)\end{array}$ & $\mathrm{p}$ for trend \\
\hline 23.0 & 33.0 & 47.5 & 67.0 & $<0.001$ \\
\hline 16.4 & 20.4 & 27.0 & 34.5 & $<0.001$ \\
\hline 7.1 & 10.7 & 15.0 & 22.7 & $<0.001$ \\
\hline 55.7 & 49.0 & 45.2 & 44.5 & $<0.001$ \\
\hline 84.0 & 84.1 & 82.9 & 79.9 & $<0.001$ \\
\hline $68.2 \pm 6.0$ & $67.4 \pm 6.1$ & $66.7 \pm 6.1$ & $65.8 \pm 6.3$ & $<0.001$ \\
\hline $74.3 \pm 11.2$ & $75.4 \pm 12.0$ & $77.4 \pm 12.0$ & $80.0 \pm 11.8$ & 0.006 \\
\hline $30.2 \pm 4.0$ & $29.9 \pm 3.9$ & $29.9 \pm 3.8$ & $29.8 \pm 3.6$ & $<0.001$ \\
\hline $210.2 \pm 223.5$ & $210.4 \pm 205.6$ & $242.9 \pm 253.7$ & $260.8 \pm 264.2$ & $<0.001$ \\
\hline $1839.3 \pm 444.5$ & $2053.8 \pm 394.2$ & $2294.2 \pm 389.2$ & $2757.4 \pm 462.4$ & $<0.001$ \\
\hline $43.1 \pm 7.2$ & $42.4 \pm 7.0$ & $41.3 \pm 6.9$ & $40.3 \pm 7.0$ & $<0.001$ \\
\hline $17.8 \pm 3.1$ & $16.9 \pm 2.6$ & $16.3 \pm 2.5$ & $15.4 \pm 2.4$ & $<0.001$ \\
\hline $38.3 \pm 7.5$ & $39.0 \pm 6.7$ & $39.7 \pm 6.5$ & $39.8 \pm 6.3$ & $<0.001$ \\
\hline $19.5 \pm 5.2$ & $19.5 \pm 4.6$ & $19.6 \pm 4.3$ & $19.4 \pm 4.0$ & 0.339 \\
\hline $5.9 \pm 2.1$ & $6.2 \pm 2.0$ & $6.4 \pm 2.1$ & $6.4 \pm 2.0$ & $<0.001$ \\
\hline $9.3 \pm 2.1$ & $9.7 \pm 2.1$ & $10.2 \pm 2.1$ & $10.7 \pm 2.3$ & $<0.001$ \\
\hline $2.2 \pm 5.2$ & $4.6 \pm 7.7$ & $8.8 \pm 11.9$ & $17.7 \pm 20.6$ & $<0.001$ \\
\hline $336.3 \pm 157.3$ & $335.0 \pm 145.1$ & $329.1 \pm 142.5$ & $335.8 \pm 143.3$ & 0.927 \\
\hline $365.0 \pm 202.9$ & $369.7 \pm 200.9$ & $371.1 \pm 199.4$ & $367.7 \pm 202.7$ & 0.355 \\
\hline $42.3 \pm 76.6$ & $78.0 \pm 126.4$ & $110.8 \pm 152.5$ & $163.8 \pm 192.2$ & $<0.001$ \\
\hline $390.5 \pm 239.6$ & $300.0 \pm 221.0$ & $243.8 \pm 206.5$ & $189.9 \pm 202.1$ & $<0.001$ \\
\hline
\end{tabular}


$\operatorname{Eggs}(\mathrm{g} / \mathrm{d})$

Red meat (g/d)

White fish $(\mathrm{g} / \mathrm{d})$

Oily fish (g/d)

Other fish and seafood $(\mathrm{g} / \mathrm{d})$

High-fat processed meat $(\mathrm{g} / \mathrm{d})$

Processed meals (g/d)

Refined grains (g/day)

Whole bread (g/d)

Chocolates (g/d)

Commercial bakery (g/d)

Sauces $(\mathrm{g} / \mathrm{d})$

Extra-virgin olive oil (g/d)

Other olive oils $(\mathrm{g} / \mathrm{d})$

Nuts $(g / d)$

$\begin{array}{ccccc}15.9 \pm 9.9 & 18.8 \pm 9.8 & 21.1 \pm 9.7 & 24.3 \pm 12.9 & <0.001 \\ 30.8 \pm 24.4 & 43.3 \pm 28.9 & 55.2 \pm 33.3 & 72.2 \pm 45.2 & <0.001 \\ 46.4 \pm 33.3 & 44.6 \pm 28.9 & 43.1 \pm 26.4 & 42.0 \pm 31.0 & <0.001 \\ 22.3 \pm 21.5 & 24.5 \pm 20.9 & 24.8 \pm 20.2 & 27.0 \pm 22.0 & <0.001 \\ 18.4 \pm 16.1 & 25.8 \pm 18.9 & 31.3 \pm 21.7 & 41.1 \pm 30.7 & <0.001 \\ 1.8 \pm 3.4 & 4.5 \pm 5.9 & 7.5 \pm 8.7 & 16.2 \pm 16.8 & <0.001 \\ 0.5 \pm 1.2 & 0.9 \pm 1.8 & 1.9 \pm 2.3 & 2.5 \pm 4.1 & <0.001 \\ 62.7 \pm 60.4 & 104.0 \pm 76.1 & 126.4 \pm 86.3 & 160.5 \pm 103.7 & <0.001 \\ 55.3 \pm 77.2 & 24.5 \pm 52.9 & 18.7 \pm 46.2 & 12.5 \pm 38.6 & <0.001 \\ 2.1 \pm 4.6 & 3.9 \pm 7.6 & 5.3 \pm 8.1 & 9.4 \pm 15.1 & <0.001 \\ 3.1 \pm 7.1 & 5.9 \pm 10.5 & 9.0 \pm 14.6 & 14.1 \pm 20.9 & <0.001 \\ 0.9 \pm 1.5 & 1.4 \pm 2.0 & 2.0 \pm 2.7 & 3.2 \pm 3.9 & <0.001 \\ 16.8 \pm 21.1 & 19.7 \pm 22.6 & 22.2 \pm 23.4 & 25.7 \pm 24.5 & <0.001 \\ 19.7 \pm 20.3 & 17.9 \pm 19.1 & 17.7 \pm 20.3 & 16.5 \pm 20.6 & 0.224 \\ 7.1 \pm 11.3 & 5.4 \pm 8.3 & 11.1 \pm 14.2 & 13.3 \pm 16.1 & <0.001\end{array}$


Table 3. Baseline characteristics of the 7216 participants according to quartiles of adherence to Mediterranean Dietary Pattern (MDP)

Men (\%)

Smoking status

Former smokers (\%)

Current smokers $(\%)$

Diabetes (\%)

Hypertension (\%)

Age (y)

Weight $(\mathrm{kg})$

BMI $\left(\mathrm{kg} / \mathrm{m}^{2}\right)$

Physical activity during leisure time (METs-min/week)

Total energy intake $(\mathrm{kcal} / \mathrm{d})$

Carbohydrate intake ( $\%$ total energy)

Protein intake ( $\%$ total energy)

Fat intake (\% total energy)

Monounsaturated fatty acid intake (\% total energy)

Polyunsaturated fatty acid intake (\% total energy)

Saturated fatty acid intake ( $\%$ total energy)

Alcohol intake (g/d)

Vegetables $(\mathrm{g} / \mathrm{d})$

Fruits (g/d)

Whole-fat dairy products $(\mathrm{g} / \mathrm{d})$

Low-fat dairy products $(\mathrm{g} / \mathrm{d})$

\begin{tabular}{|c|c|c|c|c|}
\hline $\begin{array}{c}\text { MDP1 } \\
(\mathrm{n}=1804)\end{array}$ & $\begin{array}{c}\text { MDP2 } \\
(\mathrm{n}=1804)\end{array}$ & $\begin{array}{c}\text { MDP3 } \\
(n=1804)\end{array}$ & $\begin{array}{c}\text { MDP4 } \\
(n=1804)\end{array}$ & $\mathrm{p}$ for trend \\
\hline 46.0 & 42.0 & 41.0 & 40.5 & $<0.001$ \\
\hline 22.8 & 24.5 & 24.8 & 26.3 & 0.242 \\
\hline 17.0 & 15.5 & 11.6 & 11.5 & $<0.001$ \\
\hline 50.0 & 47.1 & 48.8 & 48.7 & 0.728 \\
\hline 83.1 & 83.4 & 81.3 & 83.0 & 0.540 \\
\hline $67.6 \pm 6.4$ & $67.4 \pm 6.1$ & $66.9 \pm 6.0$ & $66.3 \pm 6.2$ & $<0.001$ \\
\hline $76.8 \pm 12.1$ & $76.8 \pm 12.1$ & $76.3 \pm 11.7$ & $76.5 \pm 12.2$ & 0.007 \\
\hline $30.2 \pm 3.7$ & $30.0 \pm 3.8$ & $29.9 \pm 3.9$ & $29.8 \pm 4.0$ & 0.001 \\
\hline $211.2 \pm 219.4$ & $224.7 \pm 247.9$ & $235.0 \pm 225.2$ & $253.2 \pm 258.9$ & $<0.001$ \\
\hline $2156.1 \pm 575.9$ & $2122.2 \pm 5239$ & $2189.7 \pm 487.5$ & $2476.6 \pm 510.9$ & $<0.001$ \\
\hline $43.2 \pm 7.5$ & $42.2 \pm 7.0$ & $41.2 \pm 6.7$ & $40.5 \pm 6.9$ & $<0.001$ \\
\hline $15.4 \pm 2.6$ & $16.6 \pm 2.7$ & $17.1 \pm 2.8$ & $17.3 \pm 2.8$ & $<0.001$ \\
\hline $38.7 \pm 7.1$ & $38.6 \pm 6.6$ & $39.4 \pm 6.6$ & $40.2 \pm 6.7$ & $<0.001$ \\
\hline $19.3 \pm 4.8$ & $19.2 \pm 4.4$ & $19.6 \pm 4.5$ & $19.9 \pm 4.5$ & $<0.001$ \\
\hline $5.9 \pm 2.2$ & $5.9 \pm 1.8$ & $6.2 \pm 1.9$ & $6.8 \pm 2.1$ & $<0.001$ \\
\hline $10.3 \pm 2.4$ & $10.0 \pm 2.2$ & $9.9 \pm 2.1$ & $9.6 \pm 2.1$ & $<0.001$ \\
\hline $9.2 \pm 16.1$ & $8.3 \pm 13.7$ & $8.1 \pm 13.8$ & $7.7 \pm 12.4$ & 0.001 \\
\hline $254.1 \pm 101.2$ & $300.4 \pm 107.9$ & $345.5 \pm 121.2$ & $436.6 \pm 178.9$ & $<0.001$ \\
\hline $285.2 \pm 156.5$ & $340.0 \pm 171.1$ & $385.8 \pm 197.8$ & $462.6 \pm 229.3$ & $<0.001$ \\
\hline $159.9 \pm 199.6$ & $94.4 \pm 141.6$ & $72.5 \pm 112.1$ & $68.2 \pm 110.2$ & $<0.001$ \\
\hline $186.9 \pm 196.0$ & $270.6 \pm 215.5$ & $312.1 \pm 231.2$ & $354.5 \pm 241.1$ & $<0.001$ \\
\hline
\end{tabular}


$\operatorname{Eggs}(\mathrm{g} / \mathrm{d})$

Red meat (g/d)

White fish $(\mathrm{g} / \mathrm{d})$

Oily fish (g/d)

Other fish and seafood $(\mathrm{g} / \mathrm{d})$

High-fat processed meat $(\mathrm{g} / \mathrm{d})$

Processed meals (g/d)

Refined grains (g/day)

Whole bread (g/d)

Chocolates (g/d)

Commercial bakery (g/d)

Sauces $(\mathrm{g} / \mathrm{d})$

Extra-virgin olive oil (g/d)

Other olive oils $(\mathrm{g} / \mathrm{d})$

Nuts $(g / d)$

$\begin{array}{ccccc}19.6 \pm 11.7 & 19.5 \pm 10.8 & 19.8 \pm 10.1 & 21.1 \pm 11.6 & <0.001 \\ 53.2 \pm 40.3 & 50.5 \pm 34.6 & 49.2 \pm 35.7 & 48.6 \pm 36.7 & <0.001 \\ 33.0 \pm 24.7 & 41.0 \pm 25.8 & 46.0 \pm 26.3 & 56.1 \pm 37.0 & <0.001 \\ 16.1 \pm 14.1 & 21.5 \pm 18.0 & 25.9 \pm 20.3 & 35.1 \pm 26.0 & <0.001 \\ 19.2 \pm 16.5 & 25.4 \pm 19.6 & 30.4 \pm 21.9 & 41.6 \pm 30.0 & <0.001 \\ 8.4 \pm 13.3 & 8.0 \pm 11.1 & 7.0 \pm 10.3 & 6.7 \pm 10.7 & <0.001 \\ 1.6 \pm 3.4 & 1.3 \pm 2.6 & 1.1 \pm 2.3 & 1.1 \pm 2.2 & 0.010 \\ 153.2 \pm 102.4 & 121.1 \pm 89.4 & 97.2 \pm 75.7 & 82.2 \pm 74.6 & <0.001 \\ 6.3 \pm 22.7 & 17.4 \pm 41.6 & 29.9 \pm 55.2 & 57.2 \pm 82.1 & <0.001 \\ 4.3 \pm 8.3 & 4.2 \pm 7.8 & 5.0 \pm 10.2 & 7.2 \pm 12.8 & <0.001 \\ 10.0 \pm 18.7 & 7.7 \pm 14.1 & 7.5 \pm 13.6 & 6.9 \pm 12.0 & <0.001 \\ 1.9 \pm 2.55 & 1.9 \pm 2.7 & 1.8 \pm 2.8 & 1.9 \pm 3.2 & 0.213 \\ 6.5 \pm 13.6 & 16.3 \pm 20.2 & 25.3 \pm 22.7 & 36.3 \pm 23.6 & <0.001 \\ 30.0 \pm 20.5 & 20.0 \pm 19.4 & 13.6 \pm 18.2 & 8.2 \pm 15.1 & <0.001 \\ 4.1 \pm 6.3 & 7.1 \pm 8.9 & 10.3 \pm 11.7 & 18.9 \pm 19.1 & <0.001\end{array}$


173 fruit. They had a statistically significant lower consumption of white fish, whole bread, and

174 low-fat dairy products. Participants with a higher score for the MDP were more likely to be

175 women, non-smokers and more physically active. They presented a reduced intake of

176 carbohydrate, and higher intake of protein and fat, specifically mono- and poly-unsaturated

177 fat.

178 Table 4 shows the hazard ratios (HR) for all-cause mortality according to baseline adherence

179 to both DP in the whole PREDIMED cohort. After adjustment for sex, age, intervention group

180 and recruitment center (model 1), there was no significant association between the upper

181 quartile of WDP and death (HR 1.07; 95\% CI: 0.77,1.50). Model 2, including additional

182 confounders, showed similar results (HR 1.04; 95\% CI: 0.74,1.47).

183 However, adherence to the empirically-derived MDP did show an inverse association that was

184 statistically significant for the linear trend $(\mathrm{p}<0.001)$ and suggested strong risk reductions in

185 the two upper quartiles, with HR $0.74(95 \% \mathrm{CI}: 0.54,0.99)$ for the third versus the first

186 quartile and HR 0.53 (95\% CI: $0.38,0.75)$ for the fourth versus the first quartile (all in model 187 2).

188 When we assessed the association between empirically derived DP and cardiovascular events

189 (myocardial infarction, stroke or cardiovascular death, i.e. the primary end-point of the main

190 trial) no significant association was found between the WDP and the risk of cardiovascular

191 events (HR for the upper versus the lowest quartile: 1.00; 95\% CI: 0.70,1.43; adjusted for sex,

192 age, intervention group and recruitment center) (table 5). Model 2, including additional

193 confounders, showed similar results (HR 1.05; 95\% CI: 0.73,1.51).

194 In contrast, a decreased risk of cardiovascular events was observed among subjects classified

195 in the third and fourth baseline quartiles of the empirically-derived MDP (HR $0.60 ; 95 \%$ CI:

196 0.42, 0.81 and HR 0.52; 95\%CI: 0.36, 0.74; respectively in model 1 and 2). Results were

197 similar 
Table 4. Hazard ratios for total mortality according to quartiles of baseline adherence to the Western dietary pattern and Mediterranean dietary pattern in the PREDIMED study with the three intervention groups considered together $(n=7216)$

\begin{tabular}{|c|c|c|c|c|c|}
\hline Western dietary pattern & $\begin{array}{c}\text { Q1 } \\
n=1804\end{array}$ & $\begin{array}{c}\text { Q2 } \\
\mathbf{n}=1804\end{array}$ & $\begin{array}{c}\text { Q3 } \\
\text { n= } 1804\end{array}$ & $\begin{array}{c}\text { Q4 } \\
\text { n=1804 }\end{array}$ & $p$ for trend \\
\hline Overall mortality (n) & 71 & 69 & 86 & 102 & \\
\hline Person-years & 7635 & 7605 & 7805 & 8034 & \\
\hline Men $(\%)$ & 23.0 & 33.0 & 47.5 & 67.0 & $<0.001$ \\
\hline Age (y) & $68.2 \pm 6.0$ & $67.4 \pm 6.1$ & $66.7 \pm 6.1$ & $65.8 \pm 6.3$ & $<0.001$ \\
\hline Multivariable model $^{1}$ & 1 (ref) & $0.94(0.67-1.32)$ & $1.09(0.78-1.51)$ & $1.07(0.77-1.50)$ & 0.53 \\
\hline Multivariable model $^{2}$ & 1 (ref) & $0.93(0.66-1.30)$ & $1.05(0.75-1.46)$ & $1.04(0.74-1.47)$ & 0.65 \\
\hline Mediterranean dietary & Q1 & Q2 & Q3 & Q4 & \\
\hline Pattern & $n=1804$ & $n=1804$ & $n=1804$ & $n=1804$ & p for trend \\
\hline Overall mortality (n) & 115 & 85 & 73 & 55 & \\
\hline Person-years & 7882 & 7613 & 7619 & 7963 & \\
\hline Men $(\%)$ & 46.0 & 42.0 & 41.0 & 40.5 & $<0.001$ \\
\hline Age (y) & $67.6 \pm 6.4$ & $67.4 \pm 6.1$ & $66.9 \pm 6.0$ & $66.3 \pm 6.2$ & $<0.001$ \\
\hline Multivariable model $^{1}$ & 1 (ref) & $0.80(0.60-1.06)$ & $0.70(0.52-0.95)^{* *}$ & $0.51(0.36-0.71)^{* *}$ & $<0.001$ \\
\hline Multivariable model $^{2}$ & 1 (ref) & $0.82(0.62-1.10)$ & $0.74(0.54-0.99)^{* *}$ & $0.53(0.38-0.75)^{* *}$ & $<0.001$ \\
\hline
\end{tabular}

${ }^{1}$ Adjusted for sex, age, recruitment center and interventional group.

${ }^{2}$ Adjusted for sex, age, recruitment center and interventional group, smoking status (never smoker, former smoker and current smoker), baseline body mass index, physical activity during leisure time, self-reported hypertension, self-reported depression, self-reported diabetes, self-reported hypercholesterolemia and education level (three categories)

$* \mathrm{p}<0.05 * * \mathrm{p}<0.001$ 
Table 5. Hazard Ratios for primary cardiovascular event according to quartiles of baseline adherence to the Western dietary pattern and Mediterranean dietary pattern in the PREDIMED study with the three intervention groups considered together $(n=7216)$

\begin{tabular}{|c|c|c|c|c|c|}
\hline Western dietary pattern & $\begin{array}{c}\text { Q1 } \\
n=1804\end{array}$ & $\begin{array}{c}\text { Q2 } \\
\mathbf{n}=1804\end{array}$ & $\begin{array}{c}\text { Q3 } \\
\mathbf{n}=1804\end{array}$ & $\begin{array}{c}\text { Q4 } \\
n=1804\end{array}$ & p for trend \\
\hline Primary cardiovascular event (n) & 67 & 61 & 65 & 84 & \\
\hline Person-years & 7627 & 7590 & 7799 & 8024 & \\
\hline Men (\%) & 23.0 & 33.0 & 47.5 & 67.0 & $<0.001$ \\
\hline Age $(y)$ & $68.2 \pm 6.0$ & $67.4 \pm 6.1$ & $66.7 \pm 6.1$ & $65.8 \pm 6.3$ & $<0.001$ \\
\hline Multivariable model ${ }^{1}$ & 1 (ref) & $0.95(0.67-1.35)$ & $0.94(0.66-1.35)$ & $1.00(0.70-1.43)$ & 0.93 \\
\hline Multivariable model ${ }^{2}$ & 1 (ref) & $0.96(0.68-1.38)$ & $0.97(0.68-1.39)$ & $1.05(0.73-1.51)$ & 0.73 \\
\hline Mediterranean dietary & Q1 & Q2 & Q3 & Q4 & \\
\hline Pattern & $n=1804$ & $n=1804$ & $n=1804$ & $n=1804$ & p for trend \\
\hline Primary cardiovascular event (n) & 104 & 70 & 54 & 49 & \\
\hline Person-years & 7881 & 7599 & 7610 & 7950 & \\
\hline Men $(\%)$ & 46.0 & 42.0 & 41.0 & 40.5 & $<0.001$ \\
\hline Age $(y)$ & $67.6 \pm 6.4$ & $67.4 \pm 6.1$ & $66.9 \pm 6.0$ & $66.3 \pm 6.2$ & $<0.001$ \\
\hline Multivariable model ${ }^{1}$ & $1(\mathrm{ref})$ & $0.75(0.55-1.02)$ & $0.58(0.41-0.81)^{* *}$ & $0.50(0.35-0.71)^{* *}$ & $<0.001$ \\
\hline Multivariable model $^{2}$ & 1 (ref) & $0.76(0.56-1.04)$ & $0.60(0.42-0.83)^{* *}$ & $0.52(0.36-0.74) * *$ & $<0.001$ \\
\hline
\end{tabular}

\footnotetext{
${ }^{1}$ Adjusted for sex, age, recruitment center and interventional group.

${ }^{2}$ Adjusted for sex, age, recruitment center and interventional group, smoking status (never smoker, former smoker and current smoker), baseline ${ }^{2}$ Adjusted for sex, age, recruitment center and interventional group, smoking status (never smoker, former smoker and current smoker), baseline body mass index, physical activity during leisure time, self-reported hypertension, self-reported depression, self-reported diabetes, self-reported hypercholesterolemia and education level (three categories) $* \mathrm{p}<0.05 * * \mathrm{p}<0.001$
} 
198 in both multivariable models ( $\mathrm{P}$ for trend $<0.001$ ), suggesting an inverse association between

199 baseline adherence to the MDP and the risk of major cardiovascular events.

200 To further analyze the association between empirically derived DP and cardiovascular events

201 we repeated the analysis using each time as outcome each of the three components of the

202 primary end-point of the trial, namely acute myocardial infarction, stroke and cardiovascular

203 death (Table 6). For each of these analyses participants with the other 2 end-points were

204 excluded. We observed that the MDP was inversely and strongly associated with the risk of

205 myocardial infarction and also with the risk of cardiovascular death (fourth quartile vs first

206 quartile: HR 0.52 ; 95\% CI: 0.36, 0.74 and HR 0.37 ; 95\% CI: 0.18, 0.76; respectively for each

207 outcome in both adjusted models 2). Moreover, we found that higher baseline WDP

208 adherence was significantly associated with a higher risk of cardiovascular death ( $\mathrm{p}$ for linear

209 trend $=0.033$ in the multiple-adjusted model). 
Table 6. a) Hazard Ratios for acute myocardial infarction according to quartiles of baseline adherence to the Western dietary pattern and

Mediterranean dietary pattern in the PREDIMED study with the three intervention groups considered together

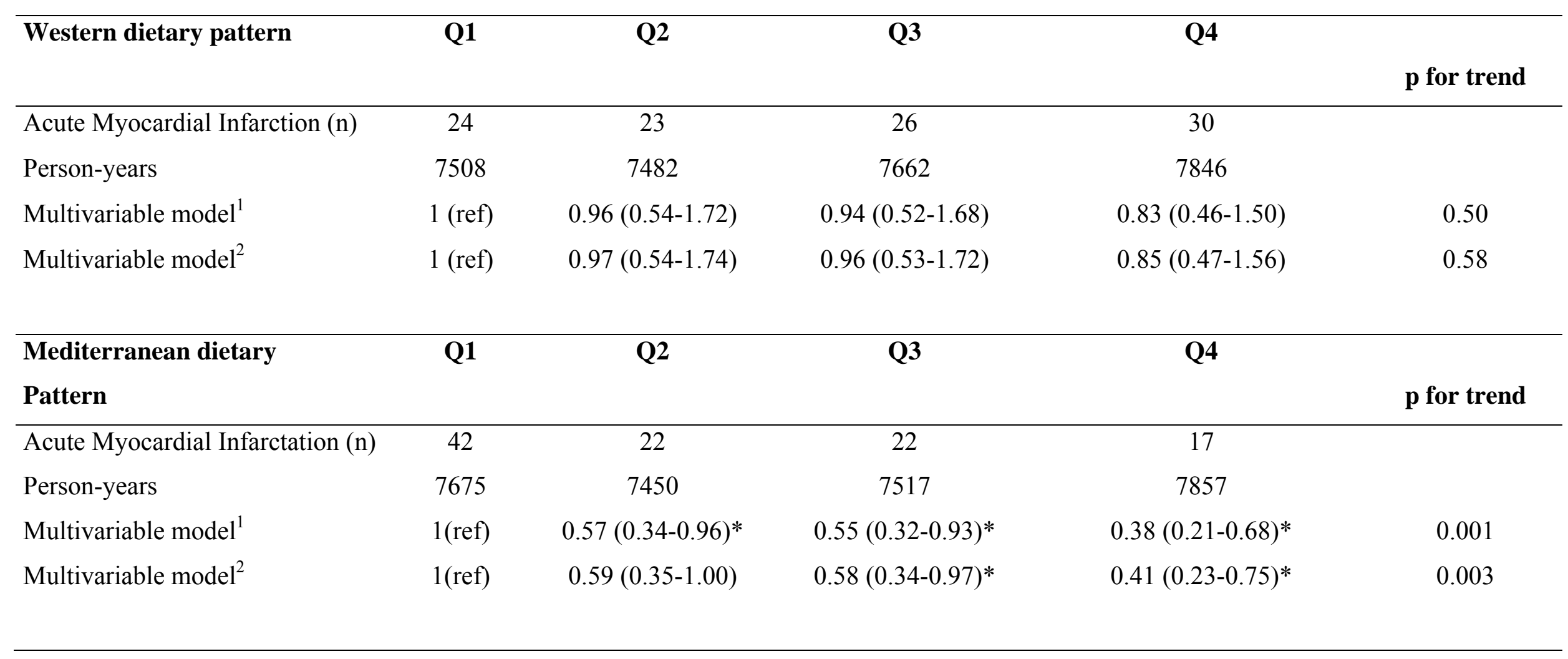


b) Hazard Ratios for stroke according to quartiles of baseline adherence to the Western dietary pattern and Mediterranean dietary pattern in the PREDIMED study with the three intervention groups considered together

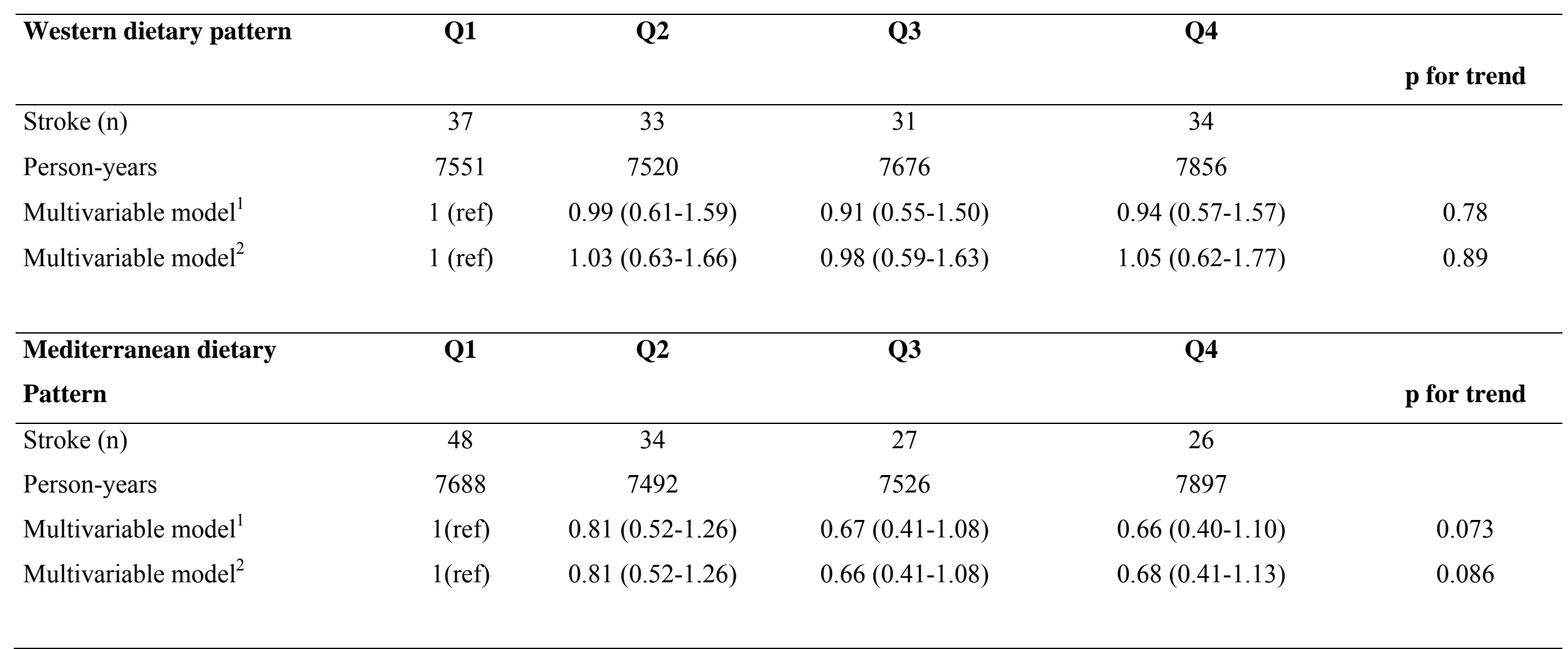


c) Hazard Ratios for cardiovascular death according to quartiles of baseline adherence to the Western dietary pattern and Mediterranean dietary pattern in the PREDIMED study with the three intervention groups considered together

\begin{tabular}{|c|c|c|c|c|c|}
\hline Western dietary pattern & Q1 & Q2 & Q3 & Q4 & $p$ for trend \\
\hline Cardiovascular death (n) & 14 & 14 & 20 & 33 & \\
\hline Multivariable model $^{1}$ & 1 (ref) & $1.03(0.48-2.19)$ & $1.41(0.69-2.85)$ & $1.90(0.95-3.77)$ & 0.031 \\
\hline Multivariable model $^{2}$ & 1 (ref) & $1.03(0.48-2.19)$ & $1.37(0.67-2.81)$ & $1.91(0.95-3.86)$ & 0.033 \\
\hline Mediterranean dietary & Q1 & Q2 & Q3 & Q4 & \\
\hline Pattern & & & & & $p$ for trend \\
\hline Cardiovascular death (n) & 32 & 23 & 15 & 11 & \\
\hline Persons-years & 7670 & 7472 & 7482 & 7857 & \\
\hline
\end{tabular}

\footnotetext{
${ }^{1}$ Adjusted for sex, age, recruitment center and interventional group.

${ }^{2}$ Adjusted for sex, age, recruitment center and interventional group, smoking status (never smoker, former smoker and current smoker), baseline ${ }^{2}$ Adjusted for sex, age, recruitment center and interventional group, smoking status (never smoker, former smoker and current smoker), baseline body mass index, physical activity during leisure time, self-reported hypertension, self-reported depression, self-reported diabetes, self-reported hypercholesterolemia and education level (three categories)

$* \mathrm{p}<0.05 * * \mathrm{p}<0.001$
} 
210 In order to assess the potential effect modification by the nutritional intervention we obtained

211 the absolute risks of death or cardiovascular events for each of the 12 groups formed by the

212 joint combination according to the nutritional intervention (3 levels) and the baseline

213 adherence to DP (quartiles). We used inverse probability weighting in this analysis to avoid

214 confounding and obtain exchangeable groups.

215 Figure 1a displays the absolute overall risk of all-cause death (rate/1000 person-year) for

216 each category made by the cross-classification according to quartiles of baseline adherence to 217 the WDP and to the 3 randomized arms of the trial (the 2 intervention groups and the control 218 group). Although statistically non-significant ( $\mathrm{p}$ for trend=0.098), a suggestion for a trend of 219 higher total mortality with higher quartiles of the WDP was present only in the control group, 220 but not in the two active intervention groups. The highest absolute risk of death was observed 221 in participants who were in the highest quartile of baseline adherence to the WDP and did not receive the intervention with MeDiet (i.e. they were allocated to the control group).

223 Likewise, figure $\mathbf{1 b}$ shows that regardless of the intervention group, better baseline adherence 224 to the MDP seemed to be associated with lower absolute risks for overall mortality in the 225 three groups, being the inverse dose-response trend statistically significant in the control and 226 in the PREDIMED active intervention group supplemented with EVOO ( $\mathrm{p}$ for trend $=0.012$ 227 and 0.027 respectively). 


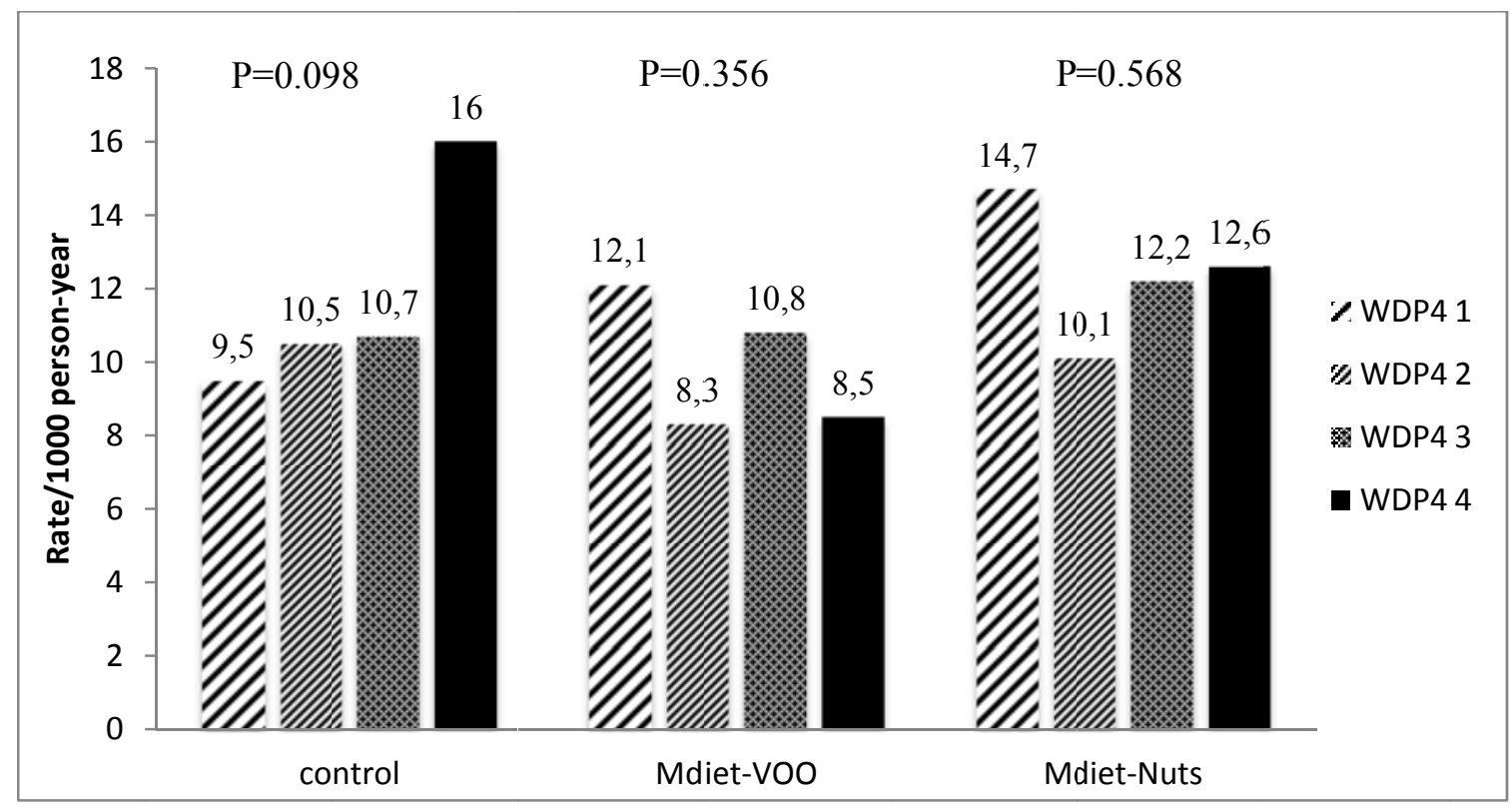

Figure 1a)

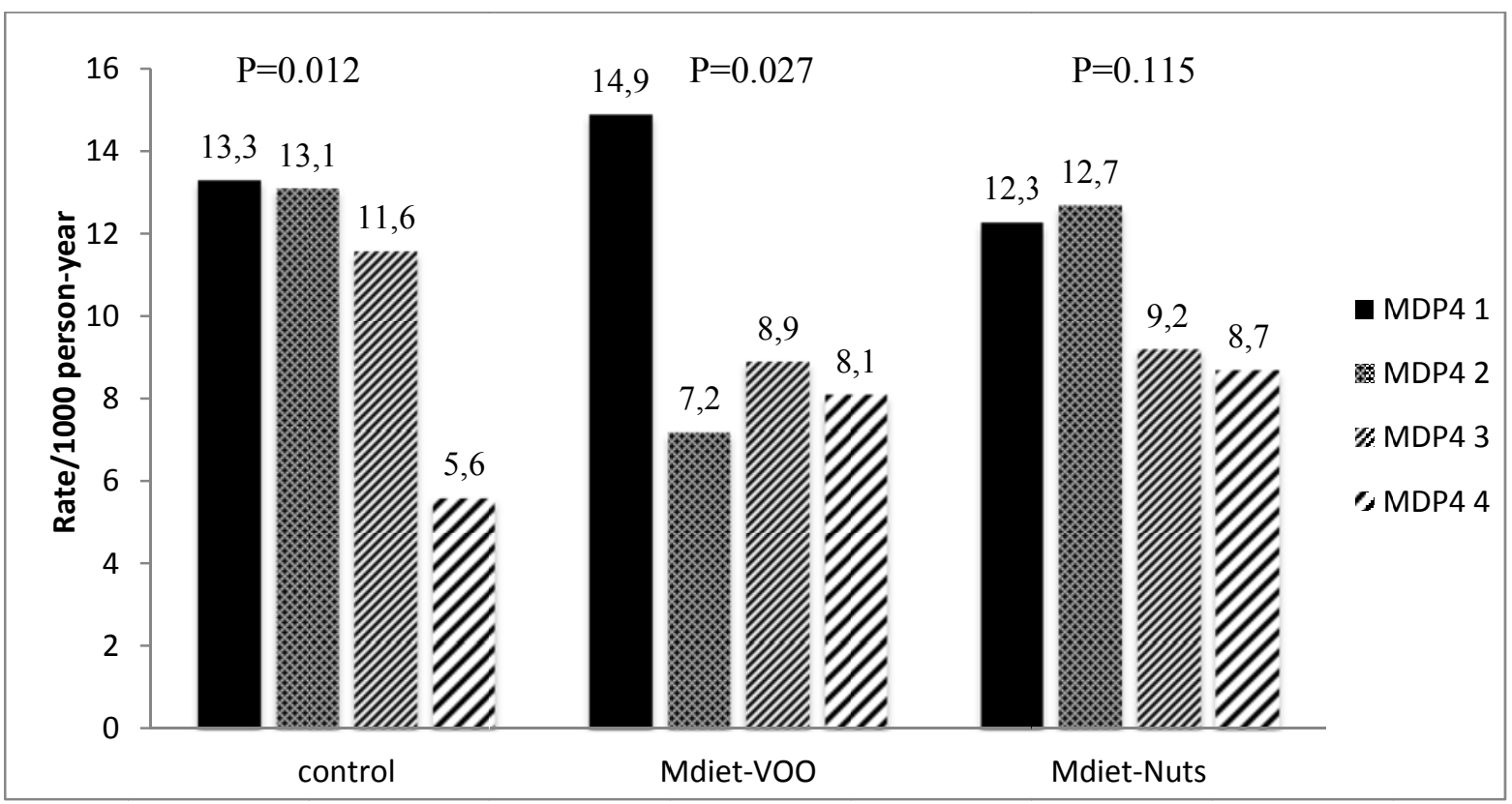

Figure 1b) 
203 Figure 2a shows the absolute all-cause mortality risk (rate/1000 person-year) for each

204 category built according to the joint classification by quartiles of WDP and allocated

205 intervention arm in the trial. No statistically significant interactions were found.

206 Finally, figure $\mathbf{2 b}$ represents the cardiovascular risk according to the joint classification by

207 both baseline adherence to the empirically-derived MDP and the allocated intervention arm.

208 These absolute rates might suggest that, even in absence of the PREDIMED nutritional

209 intervention (i.e., in the control group), the inverse association between baseline adherence to

210 MDP and cardiovascular events was clearly apparent. Interestingly, the lowest absolute rates

211 of cardiovascular events were observed in both intervention groups for the 2 upper quartiles

212 of adherence to the baseline MDP (in the group supplemented with nuts) and for the fourth

213 quartile (in the group supplemented with EVOO). The inverse dose-response trend between

214 baseline adherence to the MDP and cardiovascular events remained significant within both

215 intervention groups ( $\mathrm{p}$ for trend $=0.013$ for EVOO and 0.007 for nuts). 


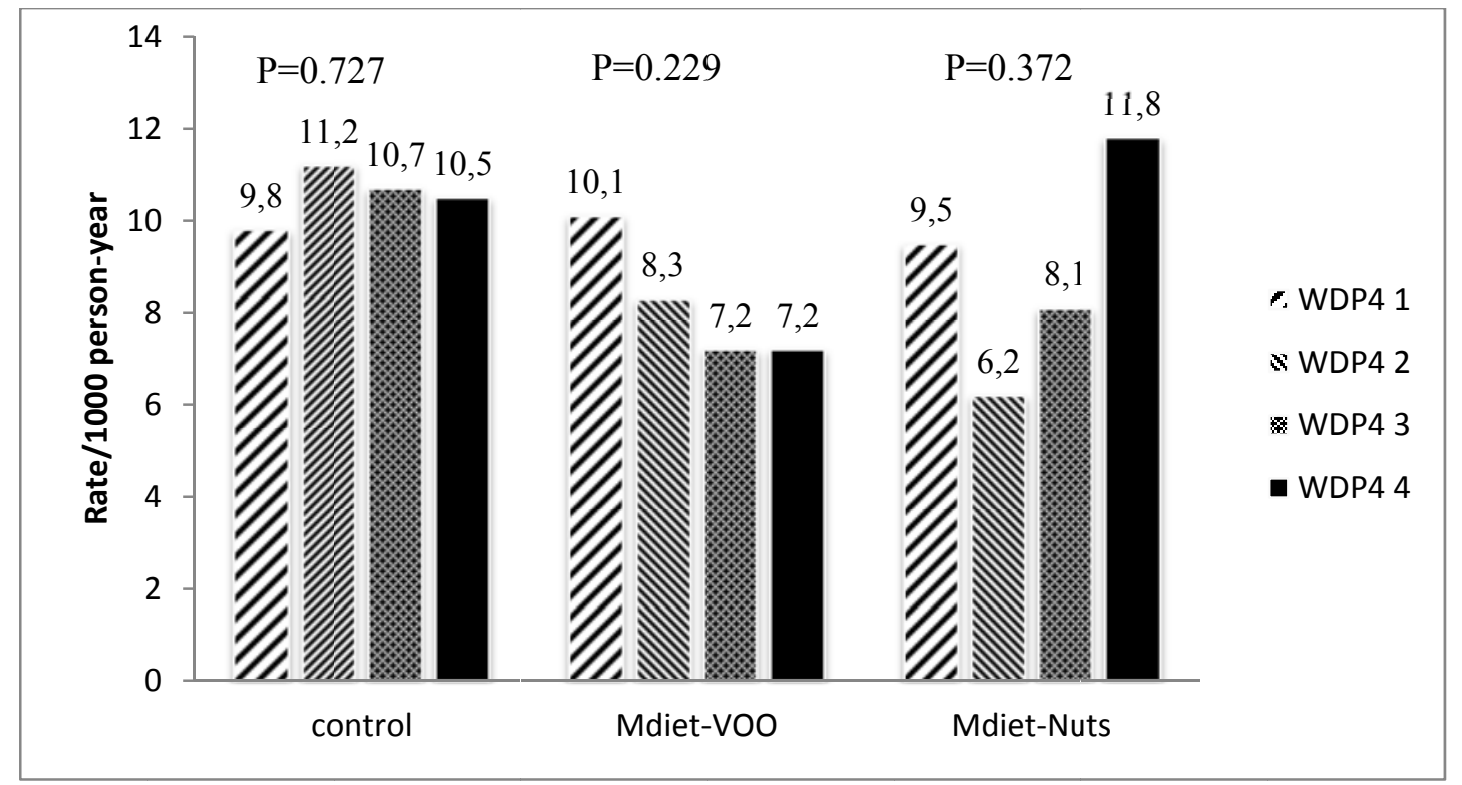

Figure 2 a)

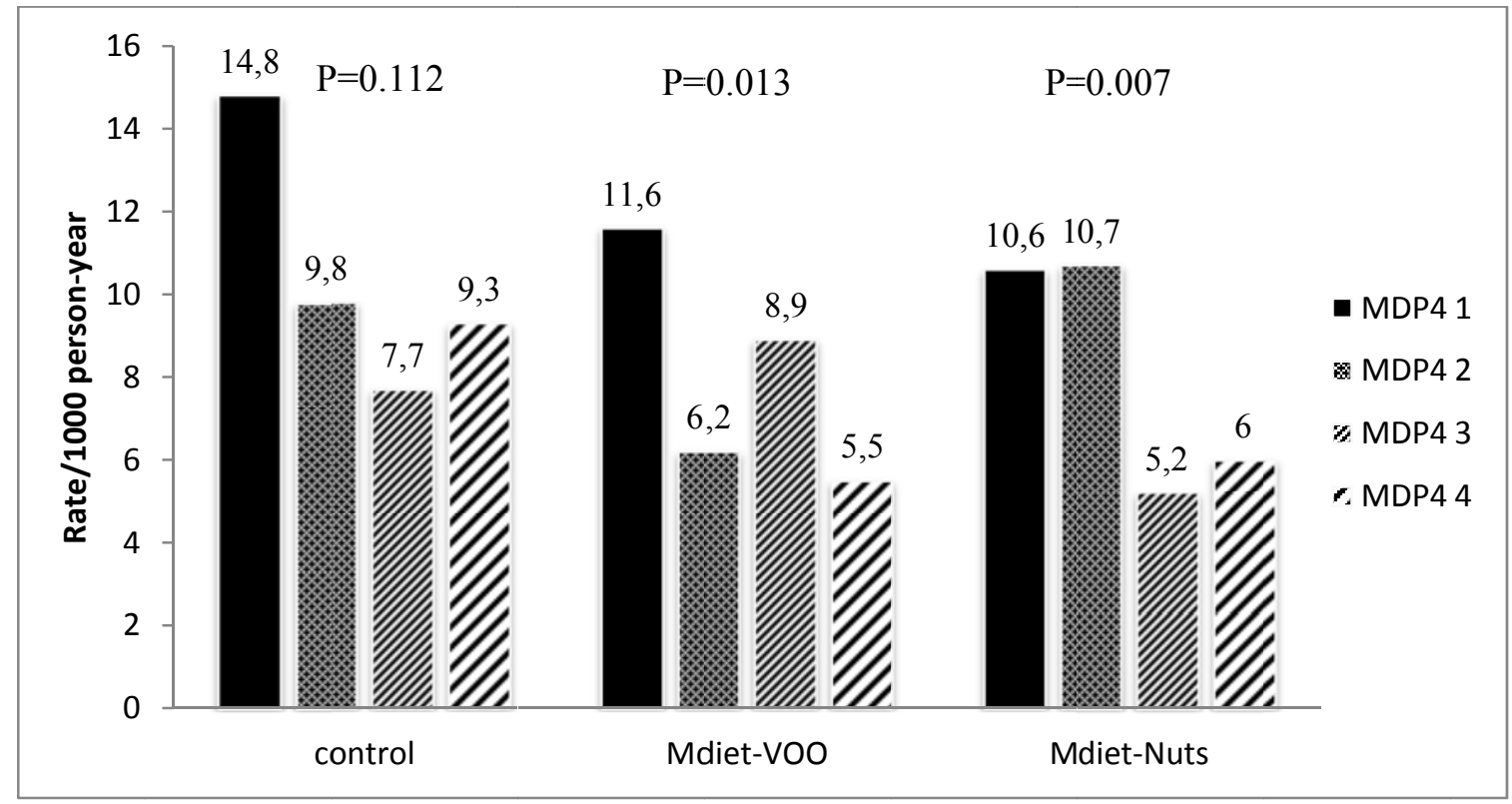

Figure 2 b) 
218 To deepen into the combined effects of baseline DP and intervention on overall mortality or

219 cardiovascular events, we assessed the potential interactions between both factors. When the

220 two intervention groups with MeDiet were merged together, we found a $p$-value $=0.054$ for the

221 interaction between the highest quartile of WDP and the MeDiet intervention in the analysis

222 of all-cause death. However, the likelihood ratio test assessing the overall effect modification

223 was not statistically significant ( 3 degrees of freedom, $p=0.27$ )

\section{DISCUSSION}

225 Using factor analysis, we investigated the association between baseline adherence to

226 empirically-derived DP (i.e. post hoc patterns) and all-cause mortality or major cardiovascular 227 events in a cohort of older Spanish subjects at high risk of CVD who underwent a nutritional 228 intervention in the PREDIMED trial $[18,19]$. Two DP were identified: WDP and MDP (table

229 1). The results showed that a closer baseline adherence to the MDP was associated with a 230 statistically significant $47 \%$ reduction in all-cause mortality along the follow-up period. An 231 inverse association of similar magnitude was also observed between the baseline adherence to 232 the MDP and cardiovascular events. These inverse associations between baseline adherence to 233 the MDP and overall mortality or cardiovascular events were always present, in a low or high 234 magnitude, regardless of the allocated intervention group. However, the intervention with the 235 MeDiet, supplemented with EVOO or nuts, in conjunction with a good baseline conformity 236 with a MDP led to the lowest absolute cardiovascular risk. On the other hand, although no 237 significant results were found, a (non-significant) suggestion for a detrimental association of 238 the WDP with a higher risk of all-cause death was present in the control group. Interestingly, 239 when specific cardiovascular events were separately analyzed, an association of the WDP 240 with a higher risk of cardiovascular death was found. Both findings suggest a detrimental 241 effect of WDP leading to a higher mortality risk. 
242 An interesting point of our study is that we were able to assess the combined effects on hard 243 clinical end-points of both the baseline adherence to empirically-derived DP and of a dietary 244 intervention. We observed that in the control group the differences between the highest and 245 lowest quartiles of both the WDP and the MDP appeared to be more apparent, especially 246 when mortality was analyzed. Control group subjects in the highest quartile of baseline 247 adherence to MDP exhibited a $42 \%$ relative reduction in their mortality risk compared to their 248 counterparts in the lowest quartile.

249 The present findings assume that the baseline DP might be a good proxy for lifetime dietary 250 exposures. In this line of thought, lifetime exposure can be more important to prevent 251 premature mortality than to follow a supplemented MeDiet or a low-fat diet for only 4 to 5 252 years. However, we were not able to demonstrate a potential interaction between a high 253 baseline adherence to WDP and the intervention with MeDiet (both active intervention groups 254 merged together versus control), though the $\mathrm{p}$ value approached the limit of statistical 255 significance $(\mathrm{p}=0.054)$.

256 Few studies have examined the association between a healthy DP using factor analysis and 257 the risk of death in high-risk elderly populations $[8,19]$. Our results agree with previous 258 reports from the Mediterranean area [8] and non-Mediterranean countries [19], where a MDP 259 was not always identified. In the same context, there are few studies analyzing empirically 260 derived DP and CVD [8, 20], and they also found that in Mediterranean areas, better 261 adherence to a MDP was associated with lower CVD risk.

262 Our results are also consistent with previous studies that have reported inverse associations 263 between an a priori defined MDP (or a DP similar to this traditional diet) and total mortality $264[19,21,22]$. In relation to CVD, there are several studies analyzing the effects of a priori defined MDP, that found a cardio-protective effect of this DP [23, 24]. However, different 
country-specific DP have been described in Mediterranean populations and these results

267 should be interpreted with caution.

268 Regarding the Spanish context, a recent study in a large cohort found that higher adherence to an empirically derived MDP in adults was associated with a reduction in the risk of all-cause mortality [6].Several explanations can account for the inverse association observed between 271 better baseline adherence to a MDP and mortality. First, the MDP has been shown to have a 272 beneficial effect on the incidence and prevalence of several diseases [25]. Second, plant-based 273 foods are protective and plant-based DP may decrease disease risk, whereas diets high in 274 animal foods may be more likely to increase the risk of mortality [26]. In fact, participants in 275 the upper baseline quartiles of MDP followed a diet rich in plant based foods and poor in 276 animal foods, and had the lowest risk of mortality. Third, the available evidence about olive 277 oil, suggests that it plays a role in the prevention of coronary heart disease, and cancer, and 278 may influence survival $[1,2,21,22,25]$. Besides, olive oil and particularly EVOO improves 279 the lipid profile and has potent antioxidant and anti- inflammatory properties $[27,28]$.

280 Though we found a WDP associated with higher cardiovascular mortality, the absence of 281 association between WDP and all-cause mortality was unexpected. Several mechanisms 282 might also be proposed to explain this absence of association. A suggested explanation is that 283 the "WDP" described in the U.S.[7] and the "WDP" in our study are not entirely equivalent 284 and may not produce the same potential adverse effects on health and longevity. Thus, the 285 consumption of foods known to be associated with lower mortality, such as fish and seafood 286 and alcohol in moderation [29], was included in the so-called WDP in our cohort. Besides, it 287 is possible that residual confounding may have affected our results.

288 Our study has several strengths, including the opportunity to assess the combined effects of 289 the baseline diet and the dietary intervention, the large sample size, the Mediterranean setting, 290 the prolonged follow-up, the sub-studies conducted to validate the questionnaire [30], and the 
objective, blinded and comprehensive ascertainment of events and close follow-up of participants.

293 There are also limitations to our study. First, the results cannot be generalized to younger 294 and/or healthier individuals from other geographical locations. Second, there is an inherent 295 difficulty to change dietary habits in elderly subjects. Third, even though we adjusted the data 296 for the main known risk factors for mortality or CVD, residual confounding cannot be 297 completely excluded. Fourth, there is a potential for measurement error in the FFQ, which 298 provides only subjective information in comparison with the use of objective markers of food 299 intake. Fifth, the number of observed deaths was small. Despite this last limitation, which is 300 associated with lower statistical power, we found a significant inverse association between the 301 MDP and total mortality. Finally, the method used to define DP (factor analysis) involves 302 several questionable decisions that must be taken into account (e.g. the definition and categorization of predefined food groups). Nevertheless, our results are in line with those of

304 other studies using similar factor analyses to define DP.

305 In conclusion, in a population of Spanish Mediterranean individuals at high cardiovascular 306 risk participating in a nutritional intervention trial, a greater baseline adherence to a MDP was 307 associated with a substantial reduction in CVD and overall mortality after follow-up for $\approx 5 \mathrm{y}$. 308 Further research is required to confirm the present findings in other Mediterranean and non309 Mediterranean settings, especially to better observe if the MeDiet could be able to reduce the 310 detrimental effect of a baseline WDP on cardiovascular or overall mortality risk. 
Acknowledgements: We thank the participants in the study and all the PREDIMED

314 personnel for the enthusiastic and sustained collaboration and all sources of support. The contribution of A. Sánchez-Villegas (University of Las Palmas. Gran Canaria) about factor

316 analysis methodology is acknowledged.

\section{Authors Contribution}

318 Conception and Design: MA Martinez-Gonzalez, I Zazpe, C Razquin.

319 Conducted research: A Sánchez-Tainta, I Zazpe and C Razquin.

320 Writing of the first draft: MA Martínez-González, C Razquin, I Zazpe, A Sánchez-Tainta.

321 Analysis and Interpretation of the Data: MA Martínez-González, C Razquin, I Zazpe.

322 Critical revision of the article for important intellectual content and final approval of the 323 article: all authors.

324 Statistical expertise: M.A. Martinez-Gonzalez and E.Toledo.

325 All authors have read and approved the final manuscript.

\section{Sources of support}

327 The supplemental foods used in the study were generously donated by Patrimonio Comunal

328 Olivarero and Hojiblanca from Spain (extra-virgin olive oil), the California Walnut

329 Commission from Sacramento, CA (walnuts) and Borges S.A. (almonds) and La Morella

330 Nuts (hazelnuts), both from Reus, Spain. CIBERobn and RTIC RD 06/0045 are initiatives of

331 ISCIII, Spain. The funding sources played no role in the design, collection, analysis, or

332 interpretation of the data or in the decision to submit the manuscript for publication.

333 Grant support: The PREDIMED trial was supported by the official funding agency for

334 Biomedical Research of the Spanish Government, Instituto de Salud Carlos III, through grants

335 provided to research networks specifically developed for the trial: RTIC G03/140

336 (Coordinator: R Estruch, MD, PhD), RTIC RD 06/0045 (Coordinator: MA Martinez-

337 Gonzalez, MD, PhD) and CIBERobn. We also acknowledge the grants from the National 
Heart Lung and Blood Institute-National Institutes of Health (1R01HL118264-01), Centro

339 Nacional de Investigaciones Cardiovasculares CNIC 06/2007, Fondo de Investigación

340 Sanitaria - Fondo Europeo de Desarrollo Regional (PI04-2239, PI05/2584, CP06/00100,

341 PI07/0240, PI07/1138, PI07/0954, PI 07/0473, PI08/1259, PI10/01407, PI11/01647,

342 PI11/01791), Ministerio de Ciencia e Innovación (AGL-2009- 13906-C02, AGL2010-22319-

343 C03), Fundación Mapfre 2010, Consejeria de Salud de la Junta de Andalucia (PI0105/2007),

344 Public Health Division of the Department of Health of the Autonomous Government of

345 Catalonia and Generalitat Valenciana (ACOMP06109, GVACOMP2010-181,

346 GVACOMP2011-151, CS2010-AP-111, CS2011-AP-042, AP-042/11 and BEST11-263) and

347 Ministerio de Economía (PI07-0954, CNIC-06, AGL2010-22319-C03-03, PI11/02505, ).

348 ET is supported by a Rio Hortega post-residency fellowship of the Instituto de Salud Carlos

349 III, Ministry of Economy and Competitiveness, Spanish Government.

Potential Financial Conflict of Interest: E. Ros received research grants from the California

Walnut Commission (Sacramento, CA) and is a nonpaid member of its Scientific Advisory

352 Committee. M.A. Martinez-Gonzalez reports received research grants from Danone and the

353 International Nut Council. J. Salas-Salvadó received research grants from the International

354 Nut Council (Reus, Spain) and is a nonpaid member of its Scientific Advisory Committee. R.

355 Estruch reports serving on the board of and receiving lecture fees from the Research

356 Foundation on Wine and Nutrition (FIVIN); serving on the boards of the Beer and Health

357 Foundation and the European Foundation for Alcohol Research (ERAB); receiving lecture

358 fees from Cerveceros de España. R.M. Lamuela-Raventos reports serving on the board of and

359 receiving lecture fees from FIVIN; receiving lecture fees from Cerveceros de España; and

360 receiving lecture fees and travel sup- port from PepsiCo. L. Serra-Majem reports serving on

361 the boards of the Mediterranean Diet Foundation and the Beer and Health Foundation. X. 
362 Pintó reports serving on the board of Omegafort; receiving lecture fees from Danone;

363 receiving payment for the development of educational presentations from Menarini; and

364 receiving grant support through his institution from Kowa, Unilever, and Karo Bio. No other 365 potential conflict of interest relevant to this article was reported.

366

367

368 


\section{References}

1. Martinez-Gonzalez MA, Bes-Rastrollo M. Dietary patterns, Mediterranean diet, and cardiovascular disease. Curr Opin Lipidol. 2014; 25: 20-26.

2. Sofi F, Macchi C, Abbate R, Gensini GF, Casini A. Mediterranean diet and health status: an updated meta-analysis and a proposal for a literature-based adherence score. Public Health Nutr. 2013; 1-14.

3. Hu FB. Dietary pattern analysis: a new direction in nutritional epidemiology. Curr Opin Lipidol. 2002; 13: 3-9.

4. Martinez-Gonzalez MA, Garcia-Lopez M, Bes-Rastrollo M, Toledo E, Martinez-Lapiscina EH, Delgado-Rodriguez M, Vazquez Z, Benito S, Beunza JJ. Mediterranean diet and the incidence of cardiovascular disease: a Spanish cohort. Nutr Metab Cardiovasc Dis. 2011; 21: 237-244.

5. Kant AK, Leitzmann MF, Park Y, Hollenbeck A, Schatzkin A. Patterns of recommended dietary behaviors predict subsequent risk of mortality in a large cohort of men and women in the United States. J Nutr. 2009; 139: 1374-1380.

6. Zazpe I, Sanchez-Tainta A, Toledo E, Sanchez-Villegas A, Martinez-Gonzalez MA. Dietary patterns and total mortality in a Mediterranean cohort: the SUN project. J Acad Nutr Diet. $2014 ; 114: 37-47$.

7. Heidemann C, Schulze MB, Franco OH, van Dam RM, Mantzoros CS, Hu FB. Dietary patterns and risk of mortality from cardiovascular disease, cancer, and all causes in a prospective cohort of women. Circulation. 2008; 118: 230-237.

8. Guallar-Castillon P, Rodriguez-Artalejo F, Tormo MJ, Sanchez MJ, Rodriguez L, Quiros JR, Navarro C, Molina E, Martinez C, Marin P, Lopez-Garcia E, Larranaga N, Huerta JM, Dorronsoro M, Chirlaque MD, Buckland G, Barricarte A, Banegas JR, Arriola L, Ardanaz E, Gonzalez CA, Moreno-Iribas C. Major dietary patterns and risk of coronary heart disease in middle-aged persons from a Mediterranean country: the EPIC-Spain cohort study. Nutr Metab Cardiovasc Dis. 2012; 22: 192-199. 
9. Anderson AL, Harris TB, Tylavsky FA, Perry SE, Houston DK, Hue TF, Strotmeyer ES, Sahyoun NR, Health ABC Study. Dietary patterns and survival of older adults. J Am Diet Assoc. 2011; 111: 84-91.

10. Hamer M, McNaughton SA, Bates CJ, Mishra GD. Dietary patterns, assessed from a weighed food record, and survival among elderly participants from the United Kingdom. Eur J Clin Nutr. 2010; 64: 853-861.

11. Masala G, Ceroti M, Pala V, Krogh V, Vineis P, Sacerdote C, Saieva C, Salvini S, Sieri S, Berrino F, Panico S, Mattiello A, Tumino R, Giurdanella MC, Bamia C, Trichopoulou A, Riboli E, Palli D. A dietary pattern rich in olive oil and raw vegetables is associated with lower mortality in Italian elderly subjects. Br J Nutr. 2007; 98: 406-415.

12. Martinez-Gonzalez MA, Corella D, Salas-Salvado J, Ros E, Covas MI, Fiol M, Warnberg J, Aros F, Ruiz-Gutierrez V, Lamuela-Raventos RM, Lapetra J, Munoz MA, Martinez JA, Saez G, Serra-Majem L, Pinto X, Mitjavila MT, Tur JA, Portillo MP, Estruch R, PREDIMED Study Investigators. Cohort profile: design and methods of the PREDIMED study. Int J Epidemiol. 2012; 41: 377-385.

13. Zazpe I, Sanchez-Tainta A, Estruch R, Lamuela-Raventos RM, Schroder H, SalasSalvado J, Corella D, Fiol M, Gomez-Gracia E, Aros F, Ros E, Ruiz-Gutierrez V, Iglesias P, Conde-Herrera M, Martinez-Gonzalez MA. A large randomized individual and group intervention conducted by registered dietitians increased adherence to Mediterranean-type diets: the PREDIMED study. J Am Diet Assoc. 2008; 108: 1134-44; discussion 1145.

14. Willet W, Stampfer S. Implications of total energy intake for epidemiologic analyses. In: Willet W, ed. Nutritional Epidemiology. New York: Oxford University Press; 1998: 273.

15. Fernandez-Ballart JD, Pinol JL, Zazpe I, Corella D, Carrasco P, Toledo E, Perez-Bauer M, Martinez-Gonzalez MA, Salas-Salvado J, Martin-Moreno JM. Relative validity of a semiquantitative food-frequency questionnaire in an elderly Mediterranean population of Spain. $\mathrm{Br}$ J Nutr. 2010; 103: 1808-1816.

16. Elosua R, Marrugat J, Molina L, Pons S, Pujol E. Validation of the Minnesota Leisure Time Physical Activity Questionnaire in Spanish men. The MARATHOM Investigators. Am J Epidemiol. 1994; 139: 1197-1209. 
17. Robins JM, Hernan MA, Brumback B. Marginal structural models and causal inference in epidemiology. Epidemiology. 2000; 11: 550-560.

18. Estruch R, Ros E, Martinez-Gonzalez MA. Mediterranean diet for primary prevention of cardiovascular disease. N Engl J Med. 2013; 369: 676-677.

19. Kant AK, Graubard BI, Schatzkin A. Dietary patterns predict mortality in a national cohort: the National Health Interview Surveys, 1987 and 1992. J Nutr. 2004; 134: 1793-1799.

20. Panagiotakos D, Pitsavos C, Chrysohoou C, Palliou K, Lentzas I, Skoumas I, Stefanadis C. Dietary patterns and 5-year incidence of cardiovascular disease: a multivariate analysis of the ATTICA study. Nutr Metab Cardiovasc Dis. 2009; 19: 253-263.

21. Tognon G, Rothenberg E, Eiben G, Sundh V, Winkvist A, Lissner L. Does the Mediterranean diet predict longevity in the elderly? A Swedish perspective. Age (Dordr). 2011; 33: 439-450.

22. Trichopoulou A, Kouris-Blazos A, Wahlqvist ML, Gnardellis C, Lagiou P, Polychronopoulos E, Vassilakou T, Lipworth L, Trichopoulos D. Diet and overall survival in elderly people. BMJ. 1995; 311: 1457-1460.

23. Kastorini CM, Milionis HJ, Kantas D, Bika E, Nikolaou V, Vemmos KN, Goudevenos JA, Panagiotakos DB. Adherence to the mediterranean diet in relation to ischemic stroke nonfatal events in nonhypercholesterolemic and hypercholesterolemic participants: results of a case/case-control study. Angiology. 2012; 63: 509-515.

24. Agnoli C, Krogh V, Grioni S, Sieri S, Palli D, Masala G, Sacerdote C, Vineis P, Tumino R, Frasca G, Pala V, Berrino F, Chiodini P, Mattiello A, Panico S. A priori-defined dietary patterns are associated with reduced risk of stroke in a large Italian cohort. J Nutr. 2011; 141: $1552-1558$

25. Sofi F, Abbate R, Gensini GF, Casini A. Accruing evidence on benefits of adherence to the Mediterranean diet on health: an updated systematic review and meta-analysis. Am J Clin Nutr. 2010; 92: 1189-1196. 
26. Orlich MJ, Singh PN, Sabate J, Jaceldo-Siegl K, Fan J, Knutsen S, Beeson WL, Fraser GE. Vegetarian dietary patterns and mortality in Adventist Health Study 2. JAMA Intern Med. 2013; 173: 1230-1238.

27. Schwingshackl L, Hoffmann G. Mediterranean dietary pattern, inflammation and endothelial function: A systematic review and meta-analysis of intervention trials. Nutr Metab Cardiovasc Dis. 2014; .

28. Cicerale S, Lucas LJ, Keast RS. Antimicrobial, antioxidant and anti-inflammatory phenolic activities in extra virgin olive oil. Curr Opin Biotechnol. 2012; 23: 129-135.

29. Gea A, Bes-Rastrollo M, Toledo E, Garcia-Lopez M, Beunza JJ, Estruch R, MartinezGonzalez MA. Mediterranean alcohol-drinking pattern and mortality in the SUN (Seguimiento Universidad de Navarra) Project: a prospective cohort study. Br J Nutr. 2014; 111: 1871-1880.

30. Schroder H, Fito M, Estruch R, Martinez-Gonzalez MA, Corella D, Salas-Salvado J, Lamuela-Raventos R, Ros E, Salaverria I, Fiol M, Lapetra J, Vinyoles E, Gomez-Gracia E, Lahoz C, Serra-Majem L, Pinto X, Ruiz-Gutierrez V, Covas MI. A short screener is valid for assessing Mediterranean diet adherence among older Spanish men and women. J Nutr. 2011; 141: 1140-1145. 


\section{Figure and table legends}

Table 1. Factor loadings for the two major dietary patterns in the PREDIMED study

Table 2. Baseline characteristics of the 7216 participants according to quartiles of adherence to Western Dietary Pattern (WDP)

Table 3. Baseline characteristics of the 7216 participants according to quartiles of adherence to Mediterranean Dietary Pattern (MDP)

Table 4. Hazard ratios for total mortality according to quartiles of baseline adherence to the Western dietary pattern and Mediterranean dietary pattern in the PREDIMED study with the three intervention groups considered together $(n=7216)$

Table 5. Hazard Ratios for primary cardiovascular event according to quartiles of baseline adherence to the Western dietary pattern and Mediterranean dietary pattern in the PREDIMED study with the three intervention groups considered together $(n=7216)$

Table 6. a) Hazard Ratios for acute myocardial infarction according to quartiles of baseline adherence to the Western dietary pattern and Mediterranean dietary pattern in the PREDIMED study with the three intervention groups considered together.

b) Hazard Ratios for stroke according to quartiles of baseline adherence to the Western dietary pattern and Mediterranean dietary pattern in the PREDIMED study with the three intervention groups considered together.

c) Hazard Ratios for cardiovascular death according to quartiles of baseline adherence to the Western dietary pattern and Mediterranean dietary pattern in the PREDIMED study with the three intervention groups considered together.

Figure 1. Absolute risks ${ }^{1}$ for overall mortality according to the quartiles of baseline adherence to WDP (a) or MDP (b) and to the randomly allocated arm of the trial (intervention groups or control group).

\footnotetext{
${ }^{1}$ Absolute risks were adjusted for potential confounders using inverse probability weighting.
} 
MDP:Mediterranean Dietary Pattern, WDP: Western Dietary Pattern.

Figure 2. Absolute risks ${ }^{2}$ for cardiovascular events according to the quartiles of baseline adherence to WDP (a) or MDP (b) and and to the randomly allocated arm of the trial (intervention groups or control group)..

MDP:Mediterranean Dietary Pattern, WDP: Western Dietary Pattern.

${ }^{2}$ Absolute risks were adjusted for potential confounders using inverse probability weighting. 


\section{Online Supporting Material}

Steering Committee - R. Estruch, D. Corella, M. Fitó, E. Ros, J. Salas-Salvado and MA Martinez-Gonzalez.

Clinical End-Point Committee - F. Aros (chair), M. Aldamiz, A. Alonso, J. Berjon, L. Forga, J. Gallego, M. A. Garcia Layana, A. Larrauri, J. Portu, J. Timiraus, and M. SerranoMartinez

\section{Other PREDIMED Investigators.}

University of Navarra, Primary Care Centers, Pamplona, Spain: B. Sanjulian, M. RuizCanela, A. Marti, JA. Martínez, M. Serrano-Martinez, J. Basterra-Gortari, E.H. MartinezLapiscina, M. Marqués, E. Goñi, Z. Vázquez.

Primary Care Centers, Servicio Navarro de Salud-Osasunbidea: J. Diez-Espino, A. GarciaArellano, N. Ortuno, N. Berrade, V. Extremera-Urabayen, C. Arroyo-Azpa, L. Garcia-Perez, T. Forcen-Alonso, P. Pascual, M. L. Garcés-Ducar, R. Martí-Massó, R. Villanueva, T. Macua, A. Parra, C.Fuertes, J. Gamboa, M.D. Martinez-Mazo, FJ Chasco Ros, A. Arillo, E. Arina. Hospital Clinic, Institut d'Investigacions Biomediques August Pi i Sunyer, Barcelona, Spain: M. Serra, A. Perez-Heras, C. Vinas, R. Casas, L. de Santamaria, S. Romero, J.M. Baena, M. Garcia, M. Oller, J. Amat, I. Duaso, Y. Garcia, C. Iglesias, C. Simon, L1. Quinzavos, L1. Parra, M. Liroz, J. Benavent, J. Clos, I. Pla, M. Amoros, M.T. Bonet, M.T. Martin, M.S. Sanchez, J. Altirruba, E. Manzano, A. Altes, M. Cofan, C. Valls, A. Sala-Vila, and M. Domenech. 
University Rovira i Virgili, Reus, Spain: M. Bullo, J. Basora, R. Gonzalez, C. Molina, F. Marquez, N. Babio, P, Martinez, N. Ibarrola, R. Balanza, M. Sorli, J. Garcia Roselló, F. Martin, R. Tort, A. Isach, B. Costa, J.J. Cabre, and J. Fernandez-Ballart.

Institute de Recerca Hospital del Mar, Barcelona, Spain: S. Tello, J. Vila, M. Fito, H.

Schroder, R. De la Torre, D. Munoz-Aguayo, R. Elosua, J. Marrugat, and M. Ferrer.

University of Valencia, Valencia, Spain: P. Carrasco, R. Osma, M. Guillen, P. Guillem-Saiz, O. Portoles, V. Pascual, C. Riera, J. Valderrama, A. Serrano, E. Lazaro, A. Sanmartin, A. Girbes, V. Santamaria, C. Sanchez and Z. Pla.

University Hospital of Alava, Vitoria, Spain: I. Salaverria, T. del Hierro, J. Algorta, S. Francisco, A. Alonso, J. San Vicente, E. Sanz, I. Felipe, A. Alonso Gomez, J. Rekondo and A. Loma-Osorio.

University of Malaga, Malaga, Spain: R. Benitez Pont, M. Bianchi Alba, J. FernandezCrehuet Navajas, J. Warnberg, R. Gomez-Huelgas, J. Martinez-Gonzalez, V. Velasco Garcia, J. de Diego Salas, A. Baca Osorio, J. Gil Zarzosa, J.J. Sanchez Luque, and E. Vargas Lopez. Instituto de la Grasa, Consejo Superior de Investigaciones Cientificas, Sevilla, Spain: J. Sanchez Perona, E. Montero Romero, A. Martin Rodriguez, and V. Martin Arranz. Institute of Health Sciences IUNICS, University of Balearic Islands, and Hospital Son Espases, Palma de Mallorca, Spain: M. Garcia-Valdueza, M. Monino, A. Proenza, R. Prieto, G. Frontera, M. Ginard, F. Fiol, A. Jover, and J. Garcia.

Department of Family Medicine, Primary Care Division of Sevilla, Sevilla, Spain: M. Leal, E. Martinez, J.M. Santos, M. Ortega-Calvo, P. Roman, F. Jose Garcia, P. Iglesias, Y. Corchado, E. Mayoral, L. Mellado, L. Miró, JM. Lozano and C. Lama. School of Pharmacy, University of Barcelona, Barcelona, Spain: M.C. Lopez- Sabater, A.I. Castellote-Bargallo, A. Medina-Remon, and A. Tresserra-Rimbau. 
University of Las Palmas de Gran Canaria, Las Palmas, Spain: J. Alvarez-Perez, A. SanchezVillegas, E. Diez Benitez, I. Bautista Castaño, I. Maldonado Diaz, M.J. Férnandez-Rodríguez, F. Sarmiendo de la Fe, C. Simon Garcia, I. Falcon Sanabria, B. Macias Gutierrez, and A.J. Santana Santana.

Hospital Universitario de Bellvitge, Hospitalet de Llobregat, Barcelona, Spain: E. de la Cruz, A. Galera, Y. Soler, F. Trias, I. Sarasa, E. Padres, and E. Corbella.

Primary Care Division, Catalan Institute of Health, Barcelona, Spain: C. Cabezas, E. Vinyoles, M.A. Rovira, L. Garcia, G. Flores, J.M. Verdu, P. Baby, A. Ramos, L. Mengual, P. Roura, M.C. Yuste, A. Guarner, A. Rovira, M.I. Santamaria, M. Mata, C. de Juan, and A. Brau.

Other investigators of the PREDIMED network: V. Ruiz-Gutierrez (Instituto de la Grasa., Sevilla), M.P. Portillo (University of Basque Country) and G. Saez (University of Valencia). 